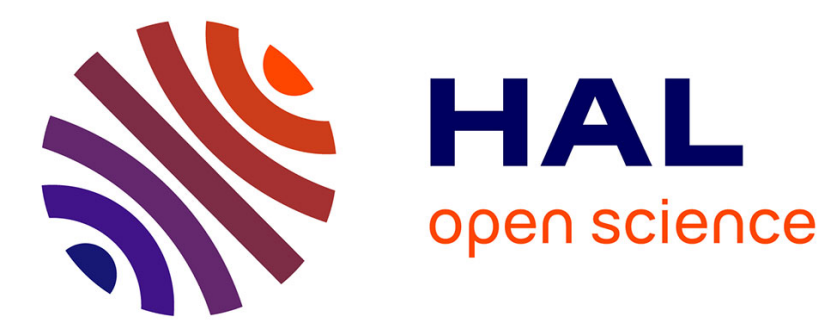

\title{
Translation on Graphs: An Isometric Shift Operator
} Benjamin Girault, Paulo Gonçalves, Eric Fleury

\section{To cite this version:}

Benjamin Girault, Paulo Gonçalves, Eric Fleury. Translation on Graphs: An Isometric Shift Operator. IEEE Signal Processing Letters, 2015, 22 (12), pp.2416 - 2420. 10.1109/LSP.2015.2488279 . hal01221562

\section{HAL Id: hal-01221562 \\ https://inria.hal.science/hal-01221562}

Submitted on 28 Oct 2015

HAL is a multi-disciplinary open access archive for the deposit and dissemination of scientific research documents, whether they are published or not. The documents may come from teaching and research institutions in France or abroad, or from public or private research centers.
L'archive ouverte pluridisciplinaire HAL, est destinée au dépôt et à la diffusion de documents scientifiques de niveau recherche, publiés ou non, émanant des établissements d'enseignement et de recherche français ou étrangers, des laboratoires publics ou privés. 


\title{
Translation on Graphs: an Isometric Shift Operator
}

\author{
Benjamin Girault, Paulo Gonçalves Member, IEEE, and Éric Fleury
}

\begin{abstract}
In this letter, we propose a new shift operator for graph signals, enforcing that our operator is isometric. Doing so, we ensure that as many properties of the time shift as possible get carried over. Finally, we show that our operator behaves reasonably for graph signals.
\end{abstract}

Index Terms-Graph signal processing, graph translation.

\section{INTRODUCTION}

Many datasets are actually structured as networks of entities, with relations of various nature between these entities. Data needing to be studied lie then on these entities. We can cite the work load in a computer network, the protein ratio in a generegulatory network, or the peer activities in a social network as examples. Such datasets are the subject of the emerging field of signal processing on graphs. Using tools borrowed and adapted from the classical signal processing toolbox, this field aims at studying these structured datasets. Whereas the subject of classical signal processing has been the study of signals supported by well defined Euclidean structures, general graphs face here the challenge of not having the mathematical comfort of the properties of Euclidean structures.

Yet, the field has seen several successes recently [14], and the toolbox of graph signal processing is growing stronger. Among the challenges not yet fully addressed, the problem of an equivalence of the time shift operator is of particular interest. Indeed, the time shift operator is at the very core of temporal signal processing, from the action of time passing by, to linear time shift invariant systems, or stationary signals defined as the time shift invariance of their stochastic properties.

Recent communications have tackled this challenge [12], [15]. Unfortunately, the shift operators defined therein are not suitable for a mathematically sound definition of stationary graph signals being statistically invariant by such operators. In this letter, we propose a novel operator and force it to be isometric. We ensure also that this operator shares key properties with the time shift. Thus, we build an isometric graph translation operator described in the spectral domain as a phase shifting operator. Finally, we describe its action using toy graph signal examples and show that it behaves reasonably in the vertex domain.

Copyright (c) 2015 IEEE. Personal use of this material is permitted. However, permission to use this material for any other purposes must be obtained from the IEEE by sending a request to pubs-permissions@ieee.org.

The authors are with the Laboratoire de l'Informatique du Parallélisme (UMR 5668: CNRS - ENS Lyon - UCB Lyon 1 - Inria), École Normale Supérieure de Lyon, Université de Lyon (firstname.lastname@ens-lyon.fr).

Work partially funded by Labex MILYON

\section{BACKGROUND}

\section{A. Time Series}

Let $x[n]=x\left(n \tau_{s}\right)$ be a time series with $\tau_{s}$ the sampling period. Let $T$ be the time shift operator such that $T\{x\}[n]=$ $x[n-1]$. This operator is at the very core of time series since its action is that of time passing by. Also, the entire field of Linear Time Shift Invariant (LTSI) filtering is dedicated to operators invariant by this time shift. Among its important properties, this operator is linear, acts as a convolution of the signal with a Kronecker's delta $\delta_{1}$ located at $n=1$, and is isometric w.r.t. the $\ell_{2}$-norm: $\|T\{x\}\|_{2}=\|x\|_{2}=\left(\sum_{n}|x[n]|^{2}\right)^{1 / 2}$.

Another central tool for time series analysis is the Fourier transform given by $X(\omega)=\sum_{n} x[n] e^{-\imath \omega n \tau_{s}}$, for any (angular) frequency $\omega$. By duality of the time sampling, $X(\omega)$ is periodic of period $\frac{2 \pi}{\tau_{s}}$, thus allowing to restrict the study of $X(\omega)$ to $\left[0, \frac{2 \pi}{\tau_{s}}\right]$. For simplicity, reduced frequencies $\widetilde{\omega}=\omega \tau_{s}$ are used in the definition of the Discrete Fourier Transform (DFT) and its inverse:

$$
\begin{aligned}
X(\widetilde{\omega}) & =\sum_{n=-\infty}^{+\infty} x[n] e^{-\imath \widetilde{\omega} n}, \quad \widetilde{\omega} \in[0,2 \pi] \\
x[n] & =\frac{1}{2 \pi} \int_{0}^{2 \pi} X(\widetilde{\omega}) e^{\imath \widetilde{\omega} n} d \widetilde{\omega} .
\end{aligned}
$$

In the analysis of $N$-periodic time series, i.e. $\left(N \tau_{s}\right)$-periodic signals, the set of reduced frequencies $\omega_{k}=2 \pi \frac{k}{N}$ with $k \in$ $\{0, \ldots, N-1\}$ is a basis of this space of periodic time series. Eq. (2) becomes:

$$
x[n]=\frac{1}{N} \sum_{k=0}^{N-1} X\left(\omega_{k}\right) e^{\imath \omega_{k} n} .
$$

In the DFT, lower reduced frequencies are close to 0 or $2 \pi$, and higher frequencies close to $\pi$. For example, the time series $x[n]=\cos \left(\frac{1}{N} n\right)$ gives $X\left(\omega_{1}\right)=X\left(\omega_{N-1}\right)=\frac{N-1}{2}$ and $X\left(\omega_{k}\right)=0$ elsewhere, coherent with $x$ being a low frequency time series.

Finally, the eigenvectors of the time shift are the Fourier modes $e_{\omega}[n]=e^{\imath \omega n}$, and its eigenvalues the complex exponentials $e^{-\imath \omega}$ such that:

$$
T\left\{e_{\omega}\right\}[n]=e^{-\imath \omega} e_{\omega}[n] .
$$

Therefore, the time shift behaves as a phase shifting operator on the Fourier modes. In this letter, we propose a new shifting operator on graph signals by analogy to these properties.

\section{B. Signal Processing on Graphs}

We start by presenting signal processing on graphs, the novel field studying signals supported by vertices of a graph. Let $\mathcal{G}=(V, E)$ be a graph with $V$ the set of vertices and 
$E \subseteq V \times V$ the set of edges between vertices. Let $N=|V|$ be the number of vertices. The adjacency matrix of the graph $\mathcal{G}$ is $A$ with $a_{i j}$ the weight of the edge $i j$ or 0 if there is no edge from $i$ to $j$. A graph signal is a function $X: V \rightarrow \mathbb{C}$ assigning a sample to each vertex. $X$ is represented as a column vector of $\mathbb{C}^{N}$, its $i^{\text {th }}$ component being the sample on the $i^{\text {th }}$ vertex.

We consider in this letter only symmetric graphs for which $i j$ is an edge if and only if $j i$ is an edge. The adjacency matrix of a symmetric graph is symmetric. We define the Laplacian matrix $L=D-A$ where $D$ is the diagonal matrix of degrees $d_{i i}=\sum_{j} a_{i j}=d_{i} . L$ is symmetric positive semidefinite [4]. $L$ has a set of orthonormal eigenvectors $\chi_{l}$ associated with nonnegative eigenvalues $\lambda_{l}$, with $L \chi_{l}=\lambda_{l} \chi_{l}$ and $0=\lambda_{0} \leq \lambda_{1} \leq$ $\cdots \leq \lambda_{N-1}$ [14]. We will suppose the graph to be connected (i.e. there is a path between any two pair of vertices in the graph) in which case $L$ has only one eigenvalue zero [4].

Performing signal processing on such a graph consists in finding a meaningful set of modes to analyse signals. Most often [14], the positive semidefinite property of the Laplacian is used to define the Fourier transform matrix $F$ as the projection on the basis of eigenvectors of $L\left\{\chi_{0}, \ldots, \chi_{N-1}\right\}$. The same property ensures that $F^{-1}=F^{*}$ and an equivalence of Parseval's identity is verified. The Laplacian matrix is then $L=F^{*} \Lambda F$, with $\Lambda$ the diagonal matrix of the eigenvalues $\lambda_{l}$.

We denote $\widehat{X}=F X$ the projection of the signal $X$ on the Fourier basis. The (generalised) convolution of two signals is then defined as a multiplication in the Fourier domain [13]:

$$
(X * Y)(i)=\sum_{l=0}^{N-1} \widehat{X}(l) \widehat{Y}(l) \chi_{l}(i) .
$$

The associated convolutive operator is $H=F^{*} \operatorname{diag}(\widehat{X}) F$ such that $H Y=X * Y$. More generally, given a linear operator $H, \widehat{H}=F H F^{*}$ denotes the expression of $H$ in the Fourier domain.

Given the complexity of the graph structure, and the properties it misses compared to Euclidean spaces, a definition of signal processing on graphs that everyone would agree on is challenging. In particular, several key tools of classical signal processing have been recently generalised to the graph structure using different approaches. Examples include the Fourier transform [14], [12], wavelet decomposition [5], [10], or the fundamental operation of translation that is at the core of temporal signal processing [12], [15]. The translation being the subject of this letter, we present two translation operators recently proposed.

1) Generalised Translations: D. Shuman, B. Ricaud \& P. Vandergheynst define the generalised translations [13] from the observation that shifting a time series by $k$ samples is equivalent to performing the convolution of the signal with a Kronecker's delta located at $k$. A generalised translation operator is then an operator $T_{k}$ such that $T_{k} X=\sqrt{N} \delta_{k} * X$. In the Fourier domain, this can be written as $\widehat{T_{k}}=\sqrt{N} \operatorname{diag}\left(\widehat{\delta_{k}}\right)$, i.e. as a diagonal matrix of diagonal $\sqrt{N} \widehat{\delta_{k}}$. These operators are linear and convolutive.

2) Graph Shift: A. Sandryhaila and J. Moura proposed to use the linear operator defined by the adjacency matrix $A$ as the equivalent of the time shift for graph signals, and call it the graph shift [12]. They define the Fourier transform as the projection on the eigenvectors of the graph shift. This definition is possible for any kind of graph, symmetric or not, and any weight on the edges. However, if $A$ is not diagonalisable, then the Jordan decomposition is used to obtain generalised eigenvectors. The graph shift is justified by analogy to signal processing on periodic time series. Indeed, periodic time series can be seen as signals on a circular oriented graph with as many vertices as samples in a period. In this context, the graph shift is exactly the time shift for $N$-periodic time series. In this letter, we only consider symmetric graphs such that $A$ is always diagonalisable. The graph shift is then an operator shifting the value on one vertex to its neighbours according to the weights of the edges.

\section{GRAPH TRANSLATION}

Both the generalised translations and the graph shift are not isometric operators with respect to the $\ell_{2}$-norm. This property being mathematically comfortable to obtain a tractable invariance property for graph signals, we propose in this section to define a new shift operator verifying it. Also, similarly to the time shift, the graph shift and the generalised translations, we define a graph translation operator that is both linear and convolutive. We will denote $T_{\mathcal{G}}$ the matrix representation of this operator and call it the graph translation. Since $T_{\mathcal{G}}$ is a convolutive operator, $\widehat{T_{\mathcal{G}}}$ is a diagonal matrix.

\section{A. Enforcing Isometry}

We design the graph translation as an isometric operator with respect to the $\ell_{2}$-norm $\|X\|_{2}=\left(\sum_{i}|X(i)|^{2}\right)^{1 / 2}$. From an energy point of view, this is equivalent to saying that the energy of the signal is left unchanged. The use of this norm follows the convention of graph signal energy found in the literature [12], [15], [1]. Since $\widehat{T_{\mathcal{G}}}$ is diagonal, isometry forces each component of this diagonal to be unimodular. Therefore, $\widehat{T_{\mathcal{G}}}=\exp (\imath \widehat{\Omega})$ with $\widehat{\Omega}$ a diagonal matrix, leading to:

$$
T_{\mathcal{G}}=\exp (\imath \Omega) \text {. }
$$

Another approach enforcing isometry would be to consider a non isometric operator $H$ and construct an isometric variant by means of output normalisation. This composed operator produces an isometric operator $H^{\text {iso }}$. However, unless $X \mapsto$ $\|H X\|_{2}$ is a constant function, it is not linear, nor does it preserve the Power Spectrum Density (PSD) $|\widehat{X}|^{2}$ of the signal.

A better approach to define $H^{\text {iso }}$ is to enforce $H^{\text {iso }}=$ $\exp (\imath \Omega)$. If $\hat{H}$ is diagonal, i.e. $H$ is a convolutive operator, and $|\widehat{H}| \leq 1$, then we can enforce $\operatorname{Re}\left(H^{\text {iso }}\right)=H$ by using $\Omega=\operatorname{acos}(H): H^{\text {iso }}=\exp (\imath \operatorname{acos}(H))$. In particular, we can isometrise any generalised translation $T_{i}^{\text {iso }}=e^{\imath \operatorname{acos}\left(\frac{1}{\sqrt{N}} T_{i}\right)}$.

\section{B. The matrix $\Omega$}

Given the general form (6), we now need to specify the matrix $\Omega$ to identify a shift operator. We propose to proceed by analogy to the temporal case to specify the expression of $T_{\mathcal{G}}$. Indeed, in the context of time series, the phase shift of a mode of frequency $\omega$ by the time shift operator is $-\omega$ as shown in (4). We build upon this observation to define $\widehat{\Omega}$. 
To define frequencies on a graph, we follow the observation in [10] in which the graph Laplacian $L$ can be seen as an approximation of the continuous Laplacian $\Delta$ (up to a change of sign). More precisely, the (temporal) Fourier modes being eigenvectors of the continuous Laplacian:

$$
\Delta e^{\imath \omega t}=-\omega^{2} e^{\imath \omega t},
$$

the eigenvalues of the continuous Laplacian are the opposite of the squared frequencies. In other words, the phase shifts are the square roots of the eigenvalues of the continuous Laplacian. This parallel can also be found in [2] where the authors prove that the graph Laplacian is an approximation of the LaplaceBeltrami operator on a manifold and that the graph Laplacian converges to the Laplace-Beltrami operator as the sampling of the manifold densifies.

Back to our graph setting, we propose to transpose this into the graph frequencies $\omega_{l}=\sqrt{\lambda_{l}},-\omega_{l}$ being then the $l^{\text {th }}$ entry of the matrix $\widehat{\Omega}$ by analogy to (4):

$$
\widehat{\Omega}=-\sqrt{\Lambda} \text {, }
$$

with $\Lambda$ the non-negative diagonal matrix of the eigenvalues of $L$. Choosing between positive and negative signs for the graph frequencies $\omega_{l}$ is an open question. In the temporal case, positive reduced frequencies lying in $[0, \pi]$ are paired with negative frequencies in $(\pi, 2 \pi)=(-\pi, 0) \bmod 2 \pi$ to produce the modes of the Fourier series. For graphs, frequencies are in general not paired by opposite frequencies, and choosing one sign or the other is a question that still needs to be elucidated. To ease the intuition, we propose in this letter to use only positive frequencies. The question of reduced frequencies is addressed in the next section.

\section{Reduced Graph Frequencies}

We saw that for time series, the Fourier transform is studied on the interval $\left[0, \frac{2 \pi}{\tau_{s}}\right]$. This interval is rescaled to match the interval $[0,2 \pi]$, defining the reduced frequencies. In this section, we propose a definition of reduced graph frequency such that they lie in the interval $[0, \pi]$, according to our choice of considering only positive frequencies.

To achieve this goal, we propose to use theoretical bounds on $\lambda_{N-1}$ proposed in the literature. The authors of [6] have proven the following upper bound (among others) on the eigenvalues of the Laplacian matrix:

$$
\rho_{\mathcal{G}}=\max _{i \in V} \sqrt{2 d_{i}\left(d_{i}+\bar{d}_{i}\right)} \quad \text { with } \quad \bar{d}_{i}=\frac{\sum_{i j \in E} w_{i j} d_{j}}{d_{i}} .
$$

This upper bound has the interesting property of being met only when the graph is bipartite regular, in other words when

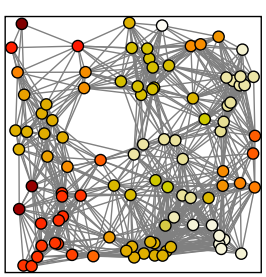

(a) $|g|(E=1)$

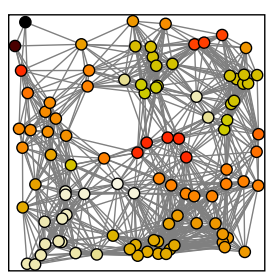

(b) $\left|T_{\mathcal{G}}^{50} g\right|(E=1)$

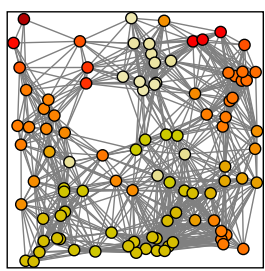

(c) $\left|T_{\mathcal{G}}^{100} g\right|(E=1)$

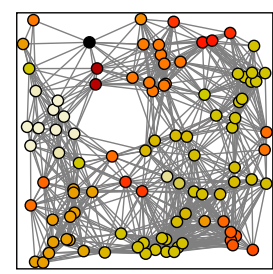

(d) $\left|T_{\mathcal{G}}^{150} g\right|(E=1)$

\begin{tabular}{|l|c|c|c|c|}
\hline & Convolution & $\begin{array}{c}\text { Mathematical } \\
\text { Group }\end{array}$ & Isometry & $\begin{array}{c}\text { Localisation } \\
\text { Invariance }\end{array}$ \\
\hline$T_{\mathcal{G}}$ & $\checkmark$ & $\checkmark$ & $\checkmark$ & $x$ \\
$A$ & $\checkmark$ & $\mathcal{V}^{*}$ & $x$ & $x$ \\
$T_{i}$ & $\checkmark$ & $x$ & $x$ & $x$ \\
\hline
\end{tabular}

* if $A$ is non singular.

the vertices of the graph can be partitioned in two sets with edges only between those two sets (bipartite), and all vertices have same degree $d_{i}=d$ (regular). In particular, the bound is met for circular graphs with even numbers of vertices, and not met for an odd number of vertices. This is similar to the DFT where the reduced frequency $\omega_{k}= \pm \pi$ exists only when $N$ is even, in which case $k=\frac{N}{2}$.

Since we have a bound on the eigenvalues of the Laplacian, we can rescale those eigenvalues such that the graph frequencies lie in the interval $[0, \pi]$ :

$$
\widetilde{\lambda}_{l}=\pi^{2} \lambda_{l} / \rho_{\mathcal{G}} \text {. }
$$

We remark that rescaling all eigenvalues of the Laplacian by the same factor is equivalent to rescaling the weights of the graph by the same factor: $U(\alpha \Lambda) U^{*}=\alpha U \Lambda U^{*}=\alpha D-$ $\alpha A$, where $\alpha=\pi^{2} / \rho_{\mathcal{G}}$. This is similar to time series where frequencies are rescaled such that they come down to the case $\tau_{s}=1$. The following definition introduces then the notion of reduced graph frequency.

Definition 1 (Reduced Graph Frequency). The reduced graph frequency associated to the Fourier mode $\chi_{l}$ is:

$$
\widetilde{\omega}_{l}=\pi \sqrt{\lambda_{l} / \rho_{\mathcal{G}}}
$$

Using this definition, we introduce our graph translation operator verifying $\widehat{T_{\mathcal{G}}}=\operatorname{diag}\left(e^{-\imath \widetilde{\omega_{0}}}, \ldots, e^{-\imath \widetilde{\omega_{N}-1}}\right)$ and such that the translated of $X$ reads $T_{\mathcal{G}} X$ :

Definition 2 (Graph Translation).

$$
T_{\mathcal{G}}=\exp \left(-\imath \pi \sqrt{L / \rho_{\mathcal{G}}}\right)
$$

\section{PROPERTIES AND Illustration}

To illustrate some properties of our graph shift operator and to compare its behaviour to that of the previously proposed forms, we use two toy signals. One is the heat kernel suggested in [15], a low-pass signal $g$ that is defined in the spectral domain as $\widehat{g}(l)=C e^{-\kappa \lambda_{l}}$, with $\|g\|_{2}=1$ (see Fig. 1(a)).

The second signal is a delta function $\delta_{i}$ located on vertex $i$, which in contrast to the previous case, comprises high frequency components. An important feature of this signal is that it allows to characterise an operator impulse response.

Fig. 1. Random graph as [15, Example 1] with $N=100, \sigma_{1}^{2}=1 / 150$ and $\sigma_{2}=0.3$. The graph translation operator is iterated on a normalised heat kernel of (a), i.e. on the signal $g$ such that $\widehat{g}\left(\lambda_{l}\right)=C e^{-50 \lambda_{l}}$. The translated signals being possibly complex, the colour scale illustrates its modulus on (a)-(e) and its phase on (f). See http://perso.ens-lyon.fr/benjamin.girault/ for more figures and animations.

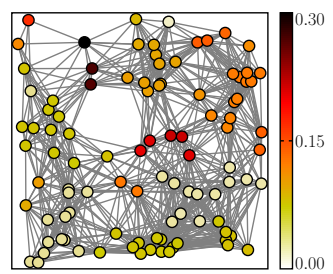

(e) $\left|T_{\mathcal{G}}^{200} g\right|(E=1)$

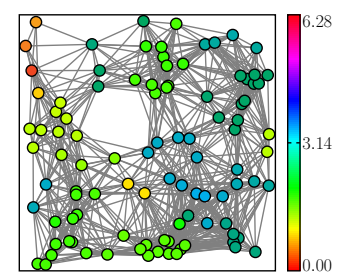

(f) $\angle\left(T_{\mathcal{G}}^{200} g\right)(E=1)$ 


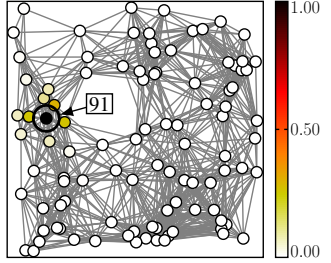

(a) $\left|T_{\mathcal{G}} \delta_{91}\right|(E=1)$

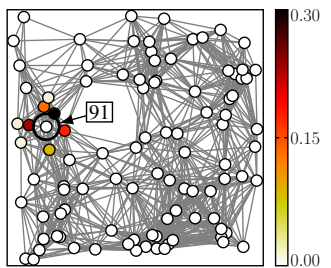

(c) $\left|A \delta_{91}\right|(E=0.44)$

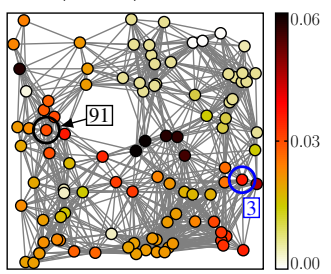

(e) $\left|T_{3} \delta_{91}\right|(E=0.23)$

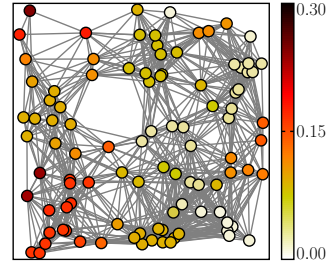

(b) $\left|T_{\mathcal{G}} g\right|(E=1)$

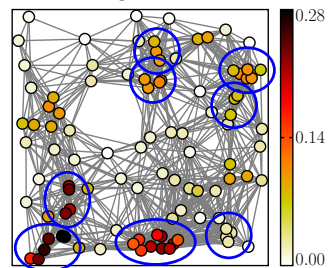

(d) $|A g|(E=1.03)$

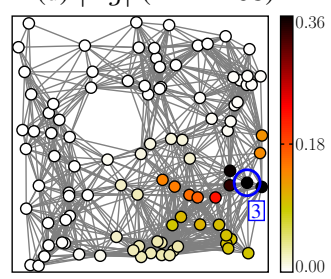

(f) $\left|T_{3} g\right|(E=0.82)$
Fig. 2. Same graph structure as Fig. 1. Various shift-like operators applied to a delta signal $\delta_{91}$ (left column) and the heat kernel $g$ of Fig. 1 (right column). On (d), the localisation of the lowest frequency components, in the sense of [11], are circled.

To ensure isometry, we introduced the graph translation in the Fourier domain as a phase shifting operator applied to the spectral components. This constraint comes at a cost, as it converts a real graph signal into a complex shifted signal. The phase of a complex graph signal is an intricate notion that still needs to be investigated (see example displayed in Fig. 1(f)), and hence we restrict the present study to the magnitude of the complex signal.

Linear convolution: All three shift operators, i.e. $T_{\mathcal{G}}, A$ and $T_{i}$, are linear convolutions, according to the definition posited in [14]. This is also the case for the usual time shift operator.

Mathematical Group: The set of translation operators $\left(T_{\mathcal{G}}^{k}\right)_{k \in \mathbb{R}}$ forms a mathematical group. This means that the iteration of $T_{\mathcal{G}}$ obeys a composition law such that $T_{\mathcal{G}}^{k} T_{\mathcal{G}}^{l}=T_{\mathcal{G}}^{k+l}$, and there exists a well-defined inverse $T_{\mathcal{G}}^{-1}$ (i.e. $T_{\mathcal{G}} T_{\mathcal{G}}^{-1}=\mathrm{Id}$ ).

This result also holds true for the graph shift operator $A$, provided the adjacency matrix $A$ is not singular. However, this is not the case for the generalised translation operator $T_{i}$ that is not, strictly speaking, a displacement operator associated to any composition law.

Isometry: Following our main motivation for introducing $T_{\mathcal{G}}$, this latter is the only shift operator that satisfies to isometry property (see Fig. 2). As a by-product, the (PSD) is invariant under graph translation: $|\widehat{T X}(l)|^{2}=|\widehat{X}(l)|^{2}, \forall l$, as it is the case with the standard time shift operator.

Localisation: An important property that one naturally expects when dealing with a shift operator is that of preserving localisation. That is, if $X$ is localised (e.g. in the sense proposed in [1, Eq. (9)]), around some origin vertex $i$, we would like it to remain localised around the target vertex $j$. Then, let us be clear: Stricto sensu, none of the proposed shift operators (neither $T_{\mathcal{G}}$ nor $A$ nor $T_{i}$ ) verify this property. It is straightforward for $A$, since it is a diffusion operator that naturally spreads out the mass located on one vertex among its neighbours (see Fig. 2(c)). The situation is more ambiguous for the generalised translation $T_{i}$. Considering the low-pass signal $g$, then $T_{i} g$ is localised on vertex $i$ (see Fig. 2(f)). Yet, $g$ itself, is not localised beforehand. Actually, the generalised convolution product that defines the action of $T_{i}$, forces the energy of $g$ to concentrate around the target vertex $i$ [14]. Conversely, applied to the Dirac mass signal $\delta_{i}$ composed of high frequency components, the localisation property breaks down and $T_{i} g$ smears out over the entire graph.

As for our proposition $T_{\mathcal{G}}$, the situation is somehow in between. The localisation property of the Dirac function $\delta_{i}$ is not preserved when shifted by $T_{\mathcal{G}}$, as illustrated in Fig. 2(a). Nonetheless, in contrast to the action of $A$, the energy of $T_{\mathcal{G}} g$ will not be split and trapped by the few vertices supporting the low frequency modes of the graph (compare Fig. 2(d) with the series of Fig. 1).

To further characterise the action of the graph translation operator, we try to get more insight about the transformation a signal undergoes when shifted by $T_{\mathcal{G}}$. To this end, we study the difference norm: $\left\|X-T_{\mathcal{G}} X\right\|_{2}^{2}=\sum_{l=0}^{N-1} 2(1-$ $\left.\cos \left(\omega_{l}\right)\right)|\widehat{X}(l)|^{2}$, which shows that spectral components of higher frequencies are more altered, whereas the DC component remains unchanged: $T_{\mathcal{G}} \chi_{0}=\chi_{0}$. This is similar to the classical time shift operator. In contrast, for the graph shift and the generalised translation, the lack of isometry prevents from drawing any conclusion from the norm of such a difference.

Tab. I summarises these properties.

\section{CONCLUSIONS}

We defined a new shift operator named the graph translation. This operator is defined in the spectral domain as an isometric operator shifting the complex phase of each of the spectral component. We saw that the requisite of being isometric is the complex nature of the operator. We focused on the modulus of translated signals to show that the graph translation behaves reasonably. Complex numbers arise regularly even when both the data and the graph can be described with real numbers and the study of the modulus is informative to study them [11], [3]. The question of the meaning of the phase of a complex graph signal still needs to be elucidated. In particular, the question of a definition of an analytic signal naturally arises in this context of complex graph signals.

Another question stems from the definition of the energy of a graph signal used to enforce the isometry of our operator. The accepted convention is to use the $\ell_{2}$-norm in both the vertex and spectral domains, but graphs are irregular structures and a norm more adapted to the structure may be better suited.

The graph translation operator we proposed in this letter allows us to propose a tractable definition of stationary graph signals. This is an on-going work, and we present and discuss its premisses in [7], [8], with an application in [9]. Finally, the theory of Linear Graph Shift Invariant filters of [12] can be adapted to our graph translation operator to obtain an equivalence of LTSI filters using the graph translation. 


\section{REFERENCES}

[1] A. Agaskar and Y. M. Lu. A Spectral Graph Uncertainty Principle. IEEE Transactions on Information Theory, 59(7):4338-4356, 2013.

[2] M. Belkin and P. Niyogi. Towards a theoretical foundation for Laplacianbased manifold methods. Journal of Computer and System Sciences, 74(8):1289-1308, 2008.

[3] C. Bordenave, M. Lelarge, and L. Massoulié. Non-backtracking spectrum of random graphs: community detection and non-regular Ramanujan graphs. arXiv preprint arXiv:1501.06087, 2015.

[4] F. R. K. Chung. Lectures on spectral graph theory. CBMS Lectures, Fresno, 1996.

[5] R. R. Coifman and M. Maggioni. Diffusion wavelets. Applied and Computational Harmonic Analysis, 21(1):53-94, 2006.

[6] K. C. Das. Extremal graph characterization from the bounds of the spectral radius of weighted graphs. Applied Mathematics and Computation, 217(18):7420-7426, 2011.

[7] B. Girault. Stationary Graph Signals using an Isometric Graph Translation. In Signal Processing Conference (EUSIPCO), 2015 Proceedings of the 23rd European. IEEE, 2015.

[8] B. Girault, P. Gonçalves, and E. Fleury. Translation and Stationarity for Graph Signals. Research Report RR-8719, École Normale Supérieure de Lyon ; Inria Rhône-Alpes, Apr. 2015.

[9] B. Girault, P. Gonçalves, and E. Fleury. Signaux Stationnaires sur graphe : étude d'un cas réel. In Proceedings of Gretsi 2015, Sept. 2015.

[10] D. K. Hammond, P. Vandergheynst, and R. Gribonval. Wavelets on graphs via spectral graph theory. Applied and Computational Harmonic Analysis, 30(2):129-150, 2011.

[11] A. Sandryhaila and J. Moura. Discrete Signal Processing on Graphs: Frequency Analysis. IEEE Transactions on Signal Processing, 62(12):3042-3054, June 2014.

[12] A. Sandryhaila and J. M. F. Moura. Discrete Signal Processing on Graphs. IEEE Transactions on Signal Processing, 61(7):1644-1656, 2013.

[13] D. Shuman, B. Ricaud, and P. Vandergheynst. A windowed graph Fourier transform. In Statistical Signal Processing Workshop (SSP), 2012 IEEE, pages 133-136. IEEE, 2012.

[14] D. I. Shuman, S. K. Narang, P. Frossard, A. Ortega, and P. Vandergheynst. The Emerging Field of Signal Processing on Graphs: Extending High-Dimensional Data Analysis to Networks and Other Irregular Domains. IEEE Signal Processing Magazine, 30(3):83-98, 2013.

[15] D. I. Shuman, B. Ricaud, and P. Vandergheynst. Vertex-frequency analysis on graphs . Applied and Computational Harmonic Analysis, 2015. in press. 